\title{
PGC and PSMA in prostate cancer diagnosis: tissue analysis from biopsy samples
}

\author{
Alberto A. Antunes, Sabrina T. Reis, Kátia R. Leite, Danilo M. Real, Juliana M. Sousa-Canavez, Luiz \\ H. Camara-Lopes, Marcos F. Dall'Oglio and Miguel Srougi \\ Laboratory of Medicine Investigation, Division of Urology, University of Sao Paulo Medical School (AAA, \\ STR, KRL, DMR, MFD, MS) and Genoa Biotechnology SA (KRL, JMS, LHC), São Paulo, Brazil
}

\section{ABSTRACT}

Purpose: The discovery of new diagnostic tools for the diagnosis of prostate cancer (PCa) has become an important field of research. In this study, we analyzed the diagnostic value of the expression of the pepsinogen $\mathrm{C}$ (PGC) and prostate-specific membrane antigen (PSMA) genes in tissue samples obtained from prostate biopsies.

Materials and Methods: This study was comprised of 51 consecutive patients who underwent transrectal ultrasound (TRUS)-guided prostate biopsies between January 2010 and March 2010. The biopsies were performed with 12 cores, and an additional core was randomly retrieved from the peripheral zone from each patient for study purposes. The expression of the PGC and PSMA genes was analyzed from the cDNA from the samples via the qRT-PCR technology. The expression patterns of patients with PCa were compared with those of patients without a PCa diagnosis.

Results: PSMA was overexpressed in only $43.4 \%$ of PCa cases, and PGC was overexpressed in $72.7 \%$ of cases. The median expression of PSMA was 1.5 times (0.1 to 43.9) and the median PGC expression was 8.7 times ( 0.1 to 50.0 ) the expression observed in prostatic tissue from TRUS-guided biopsies of normal patients. Analysis of patients with high-risk PCa indicated that PGC was overexpressed in $71.4 \%$ of cases (with a median expression of 10.6 times), and PSMA was overexpressed in only 35.7\% of cases (with a median expression of 4.5 times). Among patients with low-risk PCa, PGC was also overexpressed in $71.4 \%$ of cases (with a median expression of 5.9 times), and PSMA was overexpressed in only $42.8 \%$ of cases (with a median expression of 2.5 times).

Conclusions: PGC gene expression is significantly higher in prostatic tissue in men affected by PCa when compared to normal prostates. Further analyses are necessary to confirm our results.

\section{ARTICLE INFO}

\section{Key words:}

Prostate; Prostatic Neoplasms; Biopsy; Diagnosis;

Gene Expression; PSMA ADC

[Supplementary Concept]

Int Braz J Urol. 2013; 39: 649-56

Submitted for publication:

August 02, 2012

Accepted after revision:

April 18, 2013

\section{INTRODUCTION}

During TRUS-guided prostate biopsies, the peripheral zone is sampled in a random fashion because ultrasonography is largely unable to differentiate tumors from benign tissue. As a consequence, TRUS-guided biopsies have a high nega- tive predictive value, and $20 \%$ to $30 \%$ of patients with a negative biopsy will, in fact, exhibit cancer on subsequent biopsies (1). Additionally, significant number of tumors might also be missed because $30-40 \%$ of PCa cases are anterior or in the midline transition zone of the prostate (2). 
Researchers have sought a method that could identify cases in which TRUS leads to a false-negative result to avoid performing repeated biopsies with increased morbidity. Therefore, the development of new imaging platforms with MRI-fused images (3) and the discovery of new biological markers in serum, urine and prostatic tissue (4) have become important fields of research.

PGC is an aspartyl proteinase that is mainly involved in the digestion of proteins in the stomach. PGC is synthesized in the gastric mucosa and secreted into the gastric lumen, where it is converted into the active enzyme pepsin C under acidic conditions $(5,6)$. Surprisingly, previous studies have reported ectopic expression of PGC in several carcinomas of extragastric origin. As a type II membrane protein with folate hydrolase activity, PSMA is produced by the prostatic epithelium (7). The role of PSMA in prostate carcinogenesis is unknown, and because it is highly expressed in localized and metastatic PCa, the primary clinical applications of PSMA have been as a marker of the presence of metastatic PCa cells in the circulatory system and as a target protein for the immunotherapy of PCa (8).

We recently analyzed the expression levels of PGC and PSMA in prostatic tissue and concluded that these genes may constitute an adjunctive method to prostate biopsy in the diagnosis of PCa cases (9). The aim of the present analysis was to describe the diagnostic function of these genes using tissue samples obtained from TRUS-guided prostate biopsies.

\section{MATERIALS AND METHODS}

\section{Patients and Tumor Samples}

This study was comprised of 51 consecutive patients who underwent TRUS-guided prostate biopsies between January 2012 and March 2012. Biopsies were performed with 12 cores, and an additional core was randomly retrieved from the peripheral zone from each patient for the study purpose. These additional cores were immediately frozen at $-80^{\circ}$ C, and the 12 standard cores underwent traditional pathological analysis. Prior written and informed consent was obtained from each patient, and the study was approved by the ethics committee of the
Hospital das Clinicas from University of Sao Paulo Medical School.

Table-1 presents the patients' demographic data. Indications for TRUS-guided prostate biopsy included an altered DRE alone (one case), altered serum PSA levels alone (46 cases) and both (six cases). Patients who had undergone previous biopsies, prostatic surgeries or pelvic radiotherapy were not considered for analysis.

\section{RNA Extraction and cDNA Synthesis}

For the extraction of total RNA, frozen specimens were sectioned using a cryostat at $-30^{\circ} \mathrm{C}$ into 10 fragments of $10 \mu \mathrm{m}$. The sectioned samples were then macerated in liquid nitrogen, and total RNA was extracted with Trizol ${ }^{\circledR}$ according to a pre-established protocol (Invitrogen Life Technologies). The purity and concentration of the RNA were measured in a spectrophotometer (260/280 nM), and RNA integrity was verified in an Agilent 2100 bioanalyzer (Agilent Technologies). Synthesis of cDNA was performed from at least $5 \mu \mathrm{g}$ of total RNA with the enzyme M-MLV reverse transcriptase and random primers (Invitrogen Life Technologies, Carlsbad, California). The reactions were incubated at $65^{\circ} \mathrm{C}$ for five minutes followed by $37^{\circ} \mathrm{C}$ for one hour and, finally, $95^{\circ} \mathrm{C}$ for five minutes. The cDNA reactions were diluted to a volume of $100 \mu \mathrm{L}$ in nuclease-free water (Invitrogen Life Technologies) and were stored at $-20^{\circ} \mathrm{C}$ until further use.

\section{qRT-PCR and Gene Expression}

The expression levels of the two genes were analyzed through the qRT-PCR technology in the Abi7500 platform using the TaqMan ${ }^{\circledR}$ protocol with sample cDNA as the template. The TaqMan Endogenous Control Assay (B2M) is listed in the Appendix. The cDNA $(2 \mu \mathrm{L})$ from each tumor sample was added to a polymerase chain reaction mix containing 1x TaqMan Universal PCR Master Mix, Amp-Erase ${ }^{\circledR}$ UNG and $1 \mu \mathrm{L}$ Endogenous Control Assay or Gene Expression Assay (Applied Biosystems) in a reaction volume of $20 \mu \mathrm{L}$. The cycling conditions were $50^{\circ} \mathrm{C}$ for two minutes, $95^{\circ} \mathrm{C}$ for 10 minutes and 40 cycles of $95^{\circ} \mathrm{C}$ for 15 seconds and $60^{\circ} \mathrm{C}$ for one minute. We used the ${ }^{\Delta \Delta} \mathrm{CT}$ method to calculate the relative expression of the three target genes using 
Table 1 - Demographic characteristics of patients.

\begin{tabular}{|c|c|c|c|}
\hline Variable & $\begin{array}{l}\text { Patients with positive } \\
\text { biopsies }\end{array}$ & $\begin{array}{l}\text { Patients with negative } \\
\text { biopsies }\end{array}$ & $p$-value \\
\hline \multicolumn{4}{|l|}{ Age (years) } \\
\hline Mean & 64.26 & 63.74 & $0.838^{*}$ \\
\hline Median (min - max) & $64(44-80)$ & $64(38-79)$ & \\
\hline \multicolumn{4}{|l|}{ DRE } \\
\hline Normal (\%) & $12(41.9)$ & $14(58.1)$ & $0.225 \#$ \\
\hline Altered (\%) & $5(71.4)$ & $2(28.6)$ & \\
\hline \multicolumn{4}{|l|}{ Total PSA (ng/ml) } \\
\hline Mean & 7.94 & 6.88 & 0.561 \\
\hline Median (min - max) & $6.28(1.6-19.3)$ & $6.05(0.4-16.8)$ & \\
\hline \multicolumn{4}{|l|}{ Total PSA / Free PSA (\%) } \\
\hline Mean & 14.3 & 22.1 & 0.073 \\
\hline Median (min - max) & $12.0(0.02-0.90)$ & $18.5(0.02-0.90)$ & \\
\hline \multicolumn{4}{|c|}{ TRUS prostate volume (grams) } \\
\hline Mean & 59.59 & 59.97 & 0.804 \\
\hline Median (min - max) & $55.5(18.7-117.2)$ & $58.75(20.5-103.4)$ & \\
\hline \multicolumn{4}{|l|}{ Number of cores } \\
\hline Mean & 13.81 & 12.42 & 0.011 \\
\hline Median (min - max) & $13(12-22)$ & $12(10-18)$ & \\
\hline \multicolumn{4}{|l|}{ Gleason score } \\
\hline Mean & 6.39 & ----- & \\
\hline Median (min - max) & $6(6-8)$ & & \\
\hline
\end{tabular}

* t-Student's test

\# Fisher's exact test

Mann-Whitney test for all other

the formula ${ }^{{ }^{\Delta} \mathrm{CT}}=$ (CT target gene, $\mathrm{PC}$ sample - CT endogenous control, PC sample) - (CT target gene, BPH sample - CT endogenous control, BPH sample). The fold change in gene expression was calculated as $2^{-\Delta \Delta} \mathrm{CT}$. (CT means cycle threshold and PC means prostate cancer).

\section{Statistical analysis}

Descriptive statistics was employed to describe the characteristics of the patients, including their age, pre biopsy PSA, DRE, prostate volume, number of biopsy cores and pathological findings. 
A statistician performed all analyses for this study. All pathological findings were reviewed by a single pathologist.

The study's quantitative variables were expressed as median values and minimum and maximum values, while the qualitative variables were expressed as numbers and percentages. For the comparison of the clinical characteristics of patients with PCa, we employed the Student's t-test, Fisher's test and Mann-Whitney's test. Statistical analysis was performed using SPSS ${ }^{\circledR} 15.0$ software for Windows $^{\circledR}$, and the significance was defined as $\mathrm{p}<0.05$.

\section{RESULTS}

A PCa diagnosis was made in 23 cases. The other pathological findings included acinar atrophy (13), chronic prostatitis (6), normal prostate (8), BPH (2) and atypical small acinar proliferation (2). Analysis of biopsy samples indicated that, while PSMA was overexpressed in only $43.4 \%$ of $\mathrm{PCa}$ cases, PGC was overexpressed in $72.7 \%$ of cases. The median expression of PSMA was 0.6 times (0.1 to 43.9) $(\mathrm{p}=0.656)$ and the median PGC expression was 2.8 times ( 0.1 to 50.0$)(p=0.026)$ that of the genes' expression in prostatic tissue obtained from normal TRUS-guided biopsies (Figure-1).

According to analysis of the Gleason score, the median PGC expression levels were 2.5 (0 to 50) and 4.1 ( 0.4 to 34.4$)$ times greater among patients with a low Gleason score (6) and high scores (7 or
8), respectively, in comparison with that of patients with normal biopsies $(p=0.64)$. Analysis of PSMA expression levels also did not indicate significant differences between patients with low and high Gleason scores $(p=0.26)$. According to analysis of serum PSA levels, the median levels of PGC expression were 2.5 and 3.1 times greater among patients with low $(<10.0 \mathrm{ng} / \mathrm{ml})$ and high $(\geq 10.0 \mathrm{ng} / \mathrm{ml})$ levels, respectively $(p=0.61)$. Median PSMA expression levels also did not differ among patients with low and high PSA levels ( $p=0.54)$.

The analysis of patients with high-risk PCa, defined by a serum PSA concentration greater than $10 \mathrm{ng} / \mathrm{ml}$, a Gleason score greater than 6 or a percentage of positive biopsy cores of 50\% or greater, demonstrated that PGC was overexpressed in 71.4\% of cases (with a median expression of 3.4 times) and PSMA was overexpressed in only $35.7 \%$ of cases (with a median expression of 0.6 times) (Figure-2). Among patients with low-risk PCa, PGC was also overexpressed in $71.4 \%$ of cases (with a median expression of 1.6 times) and PSMA was overexpressed in only $42.8 \%$ of cases (with a median expression of 0.7 times) (Figure-3). No statistical difference was found between PGC expression of patients with high-risk and low-risk PCa ( $p=0.73)$.

\section{DISCUSSION}

In the present analysis of biopsy samples, we have found that PGC was overexpressed in $72.7 \%$ of

Figure 1. Quantitative expression of PSMA and PGC in tissue samples from patients with positive TRUS guided prostate biopsy.

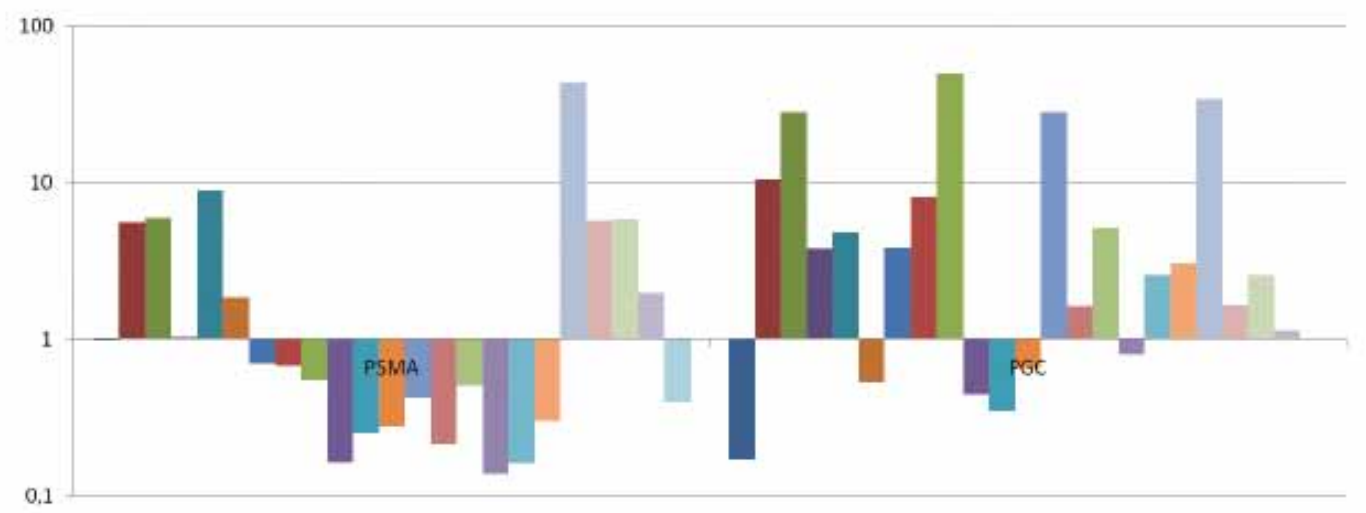


Figure 2. Quantitative expression of PSMA and PGC in biopsy samples from patients with high risk PCa.

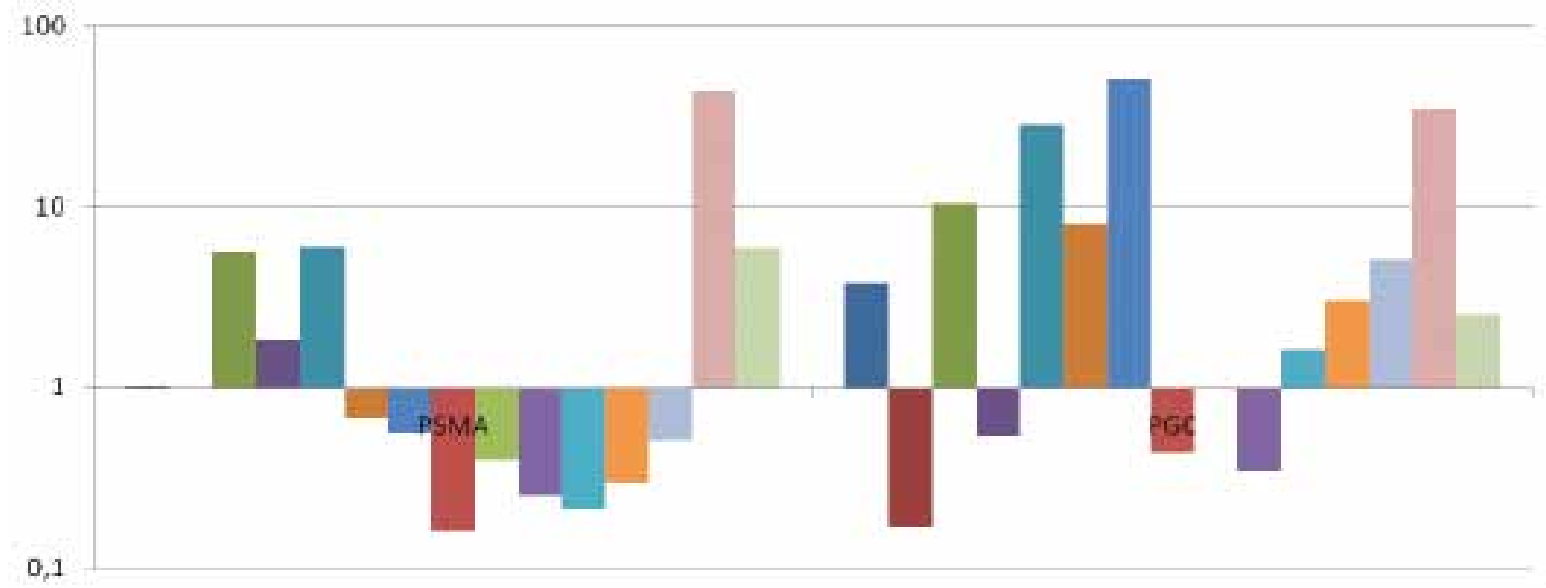

Figure 3. Quantitative expression of PSMA and PGC in prostatic tissue from patients with low risk PCa.

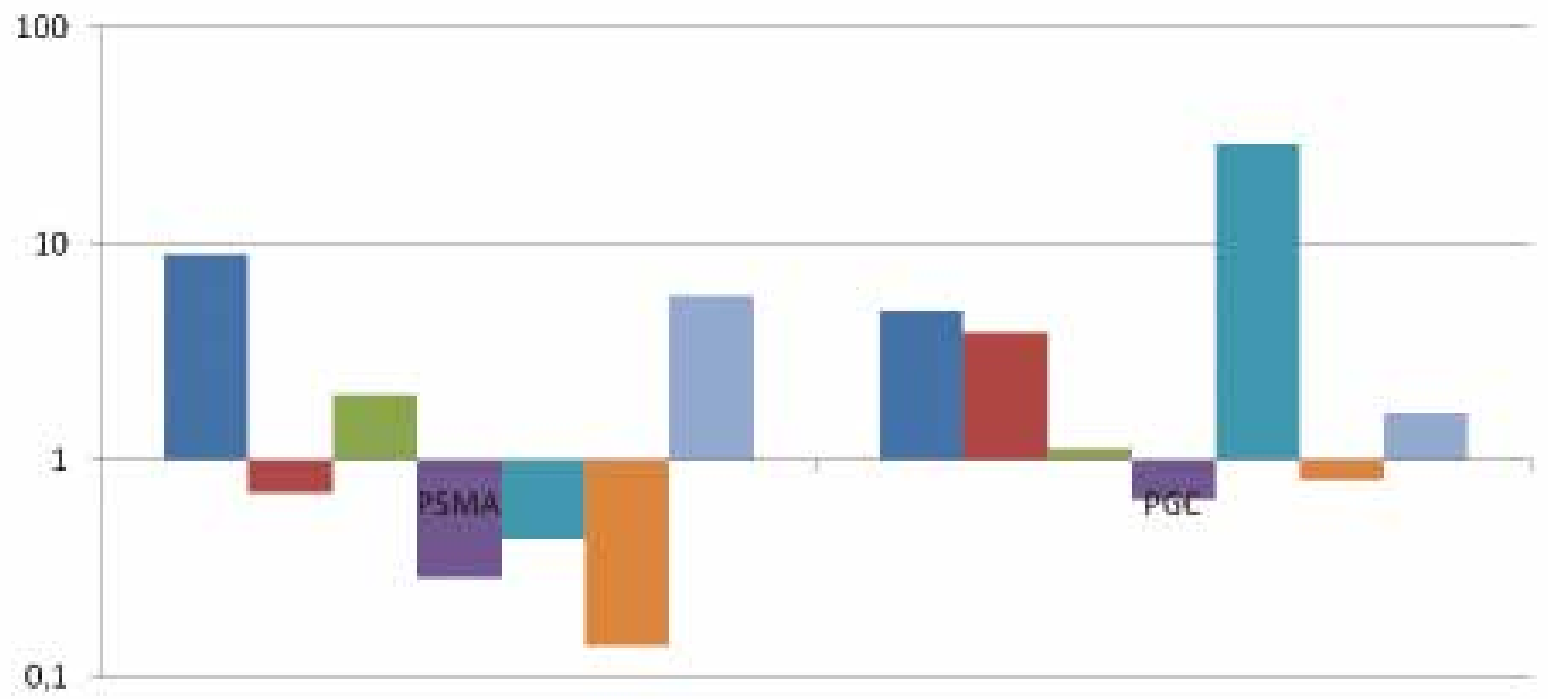

patients with PCa and exhibited an overall median expression level almost three times greater than that of patients with normal prostate biopsies. This expression pattern was retained among patients with low-risk and high-risk diseases. Quantitative analysis indicated that PGC gene expression also increased with the worsening of PCa clinical parameters with a median expression of 1.6 times and 3.4 times that of normal prostatic tissue among patients with low-risk and high-risk diseases, respectively. In contrast, PSMA was overexpressed in only $43.4 \%$ of cases with a median expression of only 0.6 times that of normal prostatic tissue.

Many studies have demonstrated that a variety of proteolytic enzymes are overproduced by cancer cells or by surrounding stromal cells from the host tissue (10). Extragastric PGC expression has been demonstrated in melanomas and cervical, 
endometrial, ovarian, pancreatic and breast carcinomas $(5,6)$. In fact, PGC has been associated with a favorable clinical outcome in breast carcinomas (11), however, the exact relevance of this gene to tumors of extragastric origin is unclear (12).

The expression of PGC in the prostate was initially described by Reese et al. (13). Through immunohistochemical analysis, they observed that, in normal conditions, PGC is found at low levels in the central zone, but not in the peripheral zone. Surprisingly, this condition changes in abnormal glands, with the expression of PGC by peripheral-zone tumors. Diamandis et al. (14) developed mouse monoclonal antibodies (Abs) against the PGC protein using highly purified antigen isolated from the gastric mucosa and employed this assay to measure PGC levels in several organic fluids. Interestingly, the highest PGC concentrations were observed in the seminal plasma, followed by breast cyst fluid and amniotic fluid. The researchers found no correlation between PSA and PGC concentrations in the serum of PCa patients and concluded that the PGC marker was not useful for either diagnosing or monitoring this malignancy.

Despite these findings, the mechanisms that control PGC expression in the prostate and the functions of the PGC protein have not been thoroughly elucidated. Because of their role in the degradation of the extracellular matrix, proteolytic enzymes are thought to be involved in the process of tumor invasion and metastasis (15). Thus, the lytic properties of PGC may indicate its function in cancer cells, given that other proteinases, such as the matrix metalloproteinase and plasminogen activators, play roles in cancer cells (10). Kohout et al. (16) investigated the proteolytic activity of PGC in normal prostate tissue, in prostate tissue exhibiting $\mathrm{BPH}$ and in prostate tissue exhibiting PCa. The PGC activity of the carcinomatous tissue did not differ significantly from the PGC activity observed in the normal prostate. In $\mathrm{BPH}$, the proteolytic activity of PGC, as assessed by the digestion of hemoglobin, was markedly reduced from that of normal prostate tissue and, when measured by the digestion of synthetic substrates, the proteolytic activity of PGC was practically nonexistent.

Additionally, for unknown reasons, PGC expression seems to be closely associated with an- drogen receptor expression in the prostate specimens of patients with PCa. In a study by Konishi et al. (17), immunohistochemical analysis of tumors obtained from radical prostatectomies demonstrated that, while androgen receptors were detected in 87\% of PGC-positive specimens, only 53\% of PGC-negative tumors expressed androgen receptors. The authors concluded that PGC might be closely related to hormonal alterations associated with the development of prostate tumors. Following this study, the prognostic role of PGC was tested in 28 patients with clinically metastatic PCa. During follow-up, disease-specific survival was 50\% for patients with PGC-negative tumors and 91\% for patients whose tumors produced the protein (12). A relationship between PGC and hormonal parameters has also been suggested to play a role in breast cancer. The expression of PGC by mammary carcinomas and cysts, but not by the normal, resting mammary gland, has led to the hypothesis that this gene may be a biochemical marker of the hormonal imbalance underlying these pathologies and may reflect the existence of cancer-associated hormonal receptor pathways $(11,18)$.

Importantly, some characteristics of the present analysis may explain the differences between the results of this study and those of our previous report. First, in the present series, the additional core retrieved for analysis was not stained with hematoxylin and eosin for the verification of tumor presence or absence. For this reason, most cores retrieved from patients with PCa diagnoses may not have exhibited adenocarcinoma within the core, and it is also possible that additional cores retrieved from patients without PCa diagnoses contained evidence of adenocarcinoma. However, considering that in our previous analysis the expression patterns of PSMA and PGC were maintained in other parts of the gland beyond the tumor area, which suggests a genetic alteration of the entire prostate (9), we did not conduct a pathological analysis of the extra cores.

Second, the control group in our previous analysis was comprised of tissue samples from the transitional zone of the prostate from patients with advanced BPH. In the present series, all additional cores (from the normal and PCa groups) were retrieved from the peripheral zone. Reese et al. (13) 
analyzed the regional distribution of PGC within the prostate through the analysis of sections obtained from the central zones, peripheral zones and seminal vesicles exposed to antibodies against PGC. Specimens were taken from 10 prostates removed during radical prostatectomies or cystectomies. Formalin-fixed tissue from the autopsy-derived prostates of four men in the third decade and from six patients with BPH nodules was also examined. They found that, in $90 \%$ of seminal vesicles and $70 \%$ of central-zone samples, more than $50 \%$ of the cells stained positive for PGC. By contrast, in 90\% of peripheral-zone samples, staining was present in 5\% or less of the epithelial cells. Similarly, PGC activity in the four autopsy prostates was observed almost entirely within the central zone and ended abruptly at the boundary between the peripheral and central zones. This condition appears to be altered in abnormal glands, with the observation of PGC expression by peripheral zone tumors (17). In our study, using the qRT-PCR technology, we detected PGC expression even in the peripheral zones of prostates without carcinoma. However, quantitative analysis indicated that median PGC expression levels were 2.8 times higher among patients with PCa diagnoses. Finally, the sample size of our present study is relatively small, which might have precluded an accurate analysis of the high-risk and low-risk PCa cases.

Some limitations of the present analysis should be pointed out. Sample size was relatively small and further analysis with a larger number of patients should be performed to confirm the results. Since this was not a randomized study, after patient selection we noted that patients with PCa diagnosis had more biopsy cores taken. Despite a statistically significant result, we don't believe that this could have influenced the results from the clinical point of view.

Finally, to confirm the actual role of these markers in the clinical practice we will perform a prospective study including patients who have a negative prostate biopsy and a rising PSA level to find out if they will have cancer in a second biopsy. Additionally, in the present series, we are going to follow the cases with a negative biopsy in which we know PGC expression to find out who is going to be diagnosed with PCa in the future.

\section{CONCLUSIONS}

PGC gene expression is significantly higher in prostatic tissue in men affected by PCa. Further analysis with a larger sample size and with the goal of determining the mechanism of function of PGC in the prostate is necessary to confirm our hypothesis.

\section{ABBREVIATIONS}

$\mathrm{PCa}=$ Prostate cancer

PSA $=$ Prostate-specific antigen

PGC = Pepsinogen $C$

PSMA = Prostate-specific membrane antigen

DRE $=$ Digital rectal examination

TRUS $=$ Transrectal ultrasound

qRT-PCR = Quantitative reverse-transcription polymerase chain reaction

$\mathrm{BPH}=$ Benign prostatic hyperplasia

RNA = Ribonucleic acid

cDNA = Complementary deoxyribonucleic acid

\section{CONFLICT OF INTEREST}

None declared.

\section{REFERENCES}

1. Djavan B, Ravery V, Zlotta A, Dobronski P, Dobrovits M, Fakhari M, et al.: Prospective evaluation of prostate cancer detected on biopsies 1, 2, 3 and 4: when should we stop? J Urol. 2001; 166: 1679-83.

2. McNeal JE, Redwine EA, Freiha FS, Stamey TA: Zonal distribution of prostatic adenocarcinoma. Correlation with histologic pattern and direction of spread. Am J Surg Pathol. 1988; 12 : 897-906.

3. Rastinehad AR, Baccala AA Jr, Chung PH, Proano JM, Kruecker J, Xu S, et al.: D'Amico risk stratification correlates with degree of suspicion of prostate cancer on multiparametric magnetic resonance imaging. J Urol. 2011; 185: 815-20.

4. Aubin SM, Reid J, Sarno MJ, Blase A, Aussie J, Rittenhouse $\mathrm{H}$, et al.: PCA3 molecular urine test for predicting repeat prostate biopsy outcome in populations at risk: validation in the placebo arm of the dutasteride REDUCE trial. J Urol. 2010; 184: 1947-52. 
5. Foltmann B: Gastric proteinases--structure, function, evolution and mechanism of action. Essays Biochem. 1981; 17: 52-84.

6. Merino AM, Vázquez J, Rodríguez JC, Fernández R, Quintela I, González LO, et al.: Pepsinogen C expression in tumors of extragastric origin. Int J Biol Markers. 2000; 15: 165-70.

7. Kinoshita Y, Kuratsukuri K, Landas S, Imaida K, Rovito PM Jr, Wang CY, et al.: Expression of prostate-specific membrane antigen in normal and malignant human tissues. World J Surg. 2006; 30: 628-36.

8. Bander NH, Milowsky MI, Nanus DM, Kostakoglu L, Vallabhajosula S, Goldsmith SJ: Phase I trial of 177lutetium-labeled J591, a monoclonal antibody to prostate-specific membrane antigen, in patients with androgen-independent prostate cancer. J Clin Oncol. 2005; 23: 4591-601.

9. Antunes AA, Leite KR, Sousa-Canavez JM, Camara-Lopes LH, Srougi $\mathrm{M}$ : The role of prostate specific membrane antigen and pepsinogen $\mathrm{C}$ tissue expression as an adjunctive method to prostate cancer diagnosis. J Urol. 2009; 181: 594-600.

10. Dos Reis ST, Pontes J Jr, Villanova FE, Borra PM, Antunes AA, Dall'oglio MF, et al.: Genetic polymorphisms of matrix metalloproteinases: susceptibility and prognostic implications for prostate cancer. J Urol. 2009; 181: 2320-5.

11. Vizoso F, Sánchez LM, Díez-Itza I, Merino AM, López-Otín C: Pepsinogen $\mathrm{C}$ is a new prognostic marker in primary breast cancer. J Clin Oncol. 1995; 13: 54-61.

12. Díaz M, Rodríguez JC, Sánchez J, Sánchez MT, Martín A, Merino $A M$, et al.: Clinical significance of pepsinogen $C$ tumor expression in patients with stage D2 prostate carcinoma. Int J Biol Markers. 2002; 17: 125-9.
13. Reese JH, McNeal JE, Redwine EA, Samloff IM, Stamey TA: Differential distribution of pepsinogen II between the zones of the human prostate and the seminal vesicle. J Urol. 1986; 136: $1148-52$.

14. Diamandis EP, Nadkarni S, Bhaumik B, Abdelrahman A, Melegos DN, Borchert G, et al.: Immunofluorometric assay of pepsinogen $\mathrm{C}$ and preliminary clinical aplications. Clin Chem. 1997; 43: 1365-71.

15. Gottesman M: The role of proteases in cancer. Semin Cancer Biol. 1990; 1: 97-160.

16. Kohout J, Kucerová Z, Korbová L, Simon V, Cypriánová A: Pepsinogen C in the human prostate. Rozhl Chir. 1989; 68: 122-7.

17. Konishi N, Nakaoka S, Matsumoto K, Nakamura M, Kuwashima S, Hiasa $Y$, et al.: Expression of pepsinogen II with androgen and estrogen receptors in human prostate carcinoma. Pathol Int. 1999; 49: 203-7.

18. Diez-Itza I, Merino AM, Tolivia J, Vizoso F, Sánchez LM, LópezOtín C: Expression of pepsinogen $\mathrm{C}$ in human breast tumours and correlation with clinicopathologic parameters. $\mathrm{Br} \mathrm{J}$ Cancer. 1993; 68: 637-40.
Correspondence address: Dr. Alberto A Antunes

Rua Barata Ribeiro, 490 / 76 São Paulo, SP, 01308-000, Brazil E-mail: antunesuro@uol.com.br 